\title{
A Collaborative Teaching Approach Using Integrated Electronic Environments for Information Security
}

\author{
Yu-An Tan, Zuo Wang, and Xu-Bo Wu \\ School of Computer Science and Engineering \\ Beijing Institute of Technology, 100081 Beijing, P.R. China \\ victortan@yeah.net, qiushui@bit.edu.cn, wuxubo@bit.edu.cn
}

\begin{abstract}
Rapid advancement and new information sources in many academic fields offer expanded opportunities for collaborative teaching. In this paper, we propose a collaborative teaching approach using integrated electronic environments for information security. Taking the security problem of implicit type conversion of $\mathrm{C}++$ program as instance, we present the learning goals \& objectives, course content, student assignments and discussion of the collaborative teaching approach using integrated electronic environments. The teaching performances suggest that this new proposed teaching approach is very efficacious.
\end{abstract}

Keywords: collaborative teaching approach; integrated electronic environments; information security; safety vulnerability.

\section{Introduction}

There is a growing call for competency-based education in educational contexts. Competencies can be construed as abilities that enable learners to recognize and define new problems in their domain of study as well as solve these problems [1]. According to Keen [2], competencies are a combination of complex cognitive and higher-order skills, highly integrated knowledge structures, interpersonal and social skills, and attitudes and values. Acquired competencies enable learners to apply these skills and attitudes in a variety of situations and over an unlimited time span [3].

In educational circles, educational designers are moving from cognitive, often rule based instructional design for efficient and effective teaching towards constructivist instructional design for competency based learning. The problem is that this is not a question of adaptation of the design methodology used, but is a question of beginning anew. For this reason, we proposed a collaborative teaching approach using integrated electronic environments for information security in this paper.

This paper is organized as following. Section 2 presents the proposed collaborative teaching approach using integrated electronic environments for information security. Finally, the conclusions of this study are drawn in section 3. 


\section{The Proposed Collaborative Teaching Approach}

In this section, we will present the proposed collaborative teaching approach using integrated electronic environments for information security. Taking the security problem of implicit type conversion of $\mathrm{C}++$ program as instance, we present the learning goals \& objectives, course content, student assignments and discussion of the collaborative teaching approach using integrated electronic environments.

\subsection{Teaching Background}

The business information plays an extremely important role in most organizations today, and efforts to protect such business information should be of the utmost importance. Information security is more often than not viewed only as a technical concern [4], and hence seems to lack the attention of top management and boards of directors. According to the current popular viewpoints, information security awareness should switch to a better program [5-6]. For this reason, the security problem of implicit type conversion of $\mathrm{C}++$ program is adopted as an instance in this paper.

\subsection{Learning Goals and Objectives}

The learning goals and objectives can be characterized as follows.

(1) Teach advanced techniques for searching data.

(2) Make students aware of information security awareness.

(3) Emphasize the importance of evaluating research findings.

(4) Provide individual assistance as necessary to ensure that all students achieve a "comfort level" result.

\subsection{Course Content}

The course contents can be described as following.

Session 1: searching technology. We will spend 10 minutes to explain the searching technology and approach, for example, Internet, BBS, library, etc.

Session 2: information security awareness. We will spend 10 minutes to explain the information security awareness. In this session, we should emphasize following two points: (1) business information plays an extremely important role today, and efforts to protect such business information should be of the utmost importance; (2) information security awareness should switch to a better program.

Session 3: The security problem of implicit type conversion of C++ program. We will spend 20 minutes to explain the security problem of implicit type conversion of $\mathrm{C}++$ program. In this session, we will apply a material example to explain this content.

\section{Conversion between Pointer \& Citing}

A pointer which points to any type object can be evaluated to the variable with the void* type. It can convert the void* type to another type with an evident way. This void* object can evaluate to that $v^{\circ}$ oid* object. Two void* objects can 
be compared each other. Besides the above basic operation, other operation to the void $^{*}$ object is insecure. In the following, we will give a material example of this kind of security problem. In this example, $p v$ points to an integer object (achieved by $p i$ in the third line). In the sixth line, this pointer was evaluated to the $p d 2$ after its conversion. As you know, this conversion is not secure.

\section{Example of a Computer Program about Conversion Between Pointers}

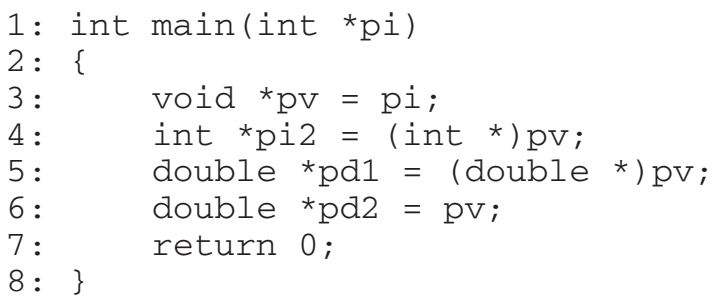

Session 4: task arrangement. We will spend 10 minutes to arrange the subsequent tasks. There are four major tasks should be finished for these students. The first one is to get together some accidents about information security. The second one is to collect some examples about the security problem of implicit type conversion in $\mathrm{C}++$ program. The third one is to design or implement some solutions for security problem of implicit type conversion in $\mathrm{C}++$ program. The forth one is to design and execute the evaluation for research findings.

Session 5: Student's presentation. We will spend 100 minutes for student's presentation. Each group of students can present their research findings with their own freeform presentation methods, but the presentation time should be limited to 20 minutes.

Session 6: Evaluating research findings. We will spend 10 minutes for evaluating research findings. The Evaluating group will estimate the presentation of each group according to their proposed index system, and they will notify the final evaluating result at this session.

\subsection{Student Assignments}

All the students were assigned as follows (Table 1).

Table 1. The student assignments

\begin{tabular}{clc}
\hline Group & \multicolumn{1}{c}{ Major Task } & Group Scale \\
\hline 1 & $\begin{array}{l}\text { Collect some accidents about information security } \\
2\end{array}$ & $\begin{array}{l}\text { Collect some examples about the security problem } \\
\text { of implicit type conversion in C++ program }\end{array}$ \\
3 & $\begin{array}{l}\text { Design or implement some solutions for security } \\
\text { problem of implicit type conversion in C++ program }\end{array}$ & $3-5$ \\
4 & Evaluate the research findings & $4-8$ \\
\hline
\end{tabular}




\subsection{Discussion}

The teaching performances of this class can be characterized as follows.

(1) Abundant teaching content. There are 3 accidents about information security, 8 examples about the security problem and 5 solutions for security problem were collected and presented by the students.

(2) Equitable evaluation for research findings. The evaluating group designed a practical and efficacious evaluation index system, and their evaluation is equitable and evenhanded on the whole.

(3) Collaborative learning approach using integrated electronic environments. All the students learned, prepared and presented their course contents with a collaborative way, and they applied many electronic types of equipment for their learning. Through this way, all the students can learn the course contents with an initiative way.

\section{Conclusions}

The contribution of this paper can be characterized as follows: it proposed a collaborative teaching approach using integrated electronic environments for information security. We present the learning goals \& objectives, course content, student assignments and discussion of the collaborative teaching approach using integrated electronic environments. The teaching performances suggest that this new proposed teaching approach is very efficacious.

\section{References}

1. Kirschner, P.A., Van Vilsteren, P., Hummel, H., et al.: A Study Environment for Acquiring Academic and Professional Competence. Studies of Higher Education, 22(2): 151-171. (1997).

2. Van Merrienboer, J.J.G.: Cognition and Multimedia Design for Complex Learning. Inaugural address available from the Educational Technology Expertise Center of the Open University of the Netherlands, (1999).

3. Keen, K.: Competence: What Is It and How Can It Be Developed? In J. Lowyck, P. de Potter, \& J. Elen (Eds.), Instructional design: Implementation issues. Brussels, Belgium: IBM International Education Center, (1992) 111-122.

4. Birman K.P.: The Next-generation Internet: Unsafe at Any Speed. IEEE Computer, 33(8): 54-60(2000).

5. Johnson E.C.: Security Awareness: Switch to a Better Programme. Network Security, 2(2): 15-18(2006).

6. Kovacich G.L.: Establishing a Network Security Programme. Computer \& Security, 15(6): 486-498(1996). 